\title{
Experimental Study to Evaluate the Significance of Musical Stimuli in Reorientation of Fetal Head Position
}

\author{
Samreen Amir ${ }^{1,3}$, Manzoor Hashmani ${ }^{2}$, Bhawany Shankar Chowdhry ${ }^{1}$, Muhammad Asif ${ }^{3}$ \\ ${ }^{1}$ Mehran University of Engineering and Technology, Jamshoro, Pakistan \\ ${ }^{2}$ Iqra University, Karachi, Pakistan \\ ${ }^{3}$ Sir Syed University of Engineering and Technology, Karachi, Pakistan
}

\begin{abstract}
The effect of music on fetal behavior and deploying music therapy to reorient the breech baby has been reported. This study aims to statistically evaluate the degree of significance of using music for such applications. It is hypothesized that the musical stimulus can elicit enough fetal response to make it change its position. Quad- instrumental is composed and applied to the mother's abdomen near the fetal head in controlled environment. The fetal basic parameters such as fetal age, fetal weight, gender, etc. are observed and the type of instrument that causes the maximum change is recorded. Out of the 46 subjects there were 32 female and 14 male fetuses. Thirteen showed displacement when stimulus was moved away from the head. The percentage of success is 28.2 hence not positive but still helpful to analyze the parametric differences between favorable and unfavorable outcomes in terms of displacement. Overall, responses of the fetuses to the given stimulus were positive and discussed. While the success rate is lower than expected, this study examined many factors that could influence the displacement results. The experiment results do not support the hypothesis that musical instruments can consistently elicit head movements from fetuses.
\end{abstract}

\section{Keywords}

Quad- instrumental, fetal response, FHR, breech presentation, music therapy, reorient

\section{INTRODUCTION}

Auditory organs of fetus are completely developed and functional at 28 weeks of gestational age. Hepper and Sara [1] reported the auditory responses of fetus at different ages and found that almost every normal fetus of 28 weeks and above responded to their audio stimulus. The frequency range and the intensity of the stimulus required to elicit the response are influenced by the gestational age. Usually a $10 \%$ reduction in intensity can elicit a response in a fetus of 38 week or above than a 28 weeks fetus when stimulated with same frequency signal. During the early stages of pregnancy most fetuses responded to frequency ranging from $400 \mathrm{~Hz}$ to $700 \mathrm{~Hz}$. As they grow up they become more sensitive to higher frequencies ranging from $1000 \mathrm{~Hz}$ to $2000 \mathrm{~Hz}$ [1], resembling auditory profiles of adults. This range also covers the human speech signal. It is assumed that the fetus can recognized the mothers, fathers and the sibling voices as soon as it is born; the strongest sound stimulus is that produced by the mother's speech [2]. It is also found in previous literature that the signal reaching the baby in womb through mother's auditory organs can also be recognized by the fetus. Behaviors such as being irritated, annoyed or pleased by some music composition heard by the mother are also reported in video documentaries showing recorded responses using 3D ultra sounds [In the womb].
The fetal heart rate is a very important parameter to observe when measuring the fetal response as a result of a given stimulus. Computerized cardiotocography analysis of FHR to acoustic stimulus was done by Nomura RM [3]. A similar study with vibrio-acoustical stimulus [4-6] has also been carried out. FHR may also vary naturally; movement, sleeping and other activities can cause the change. Gagnon [7] reported that external vibratory acoustic stimulus may cause a change from a state of sleep or rest to one of wakefulness. Usually by the end of pregnancy fetus can have a heart rate of $110-180$ beats per minute depending upon the state it is in.

As the baby reaches closely to its full size in the eighth month, that formerly spacious womb becomes a confined closet. Acrobatic movements are not likely to occur but a continuous wriggling and turning, with a few stabs of elbows and knees thrown in, may be felt. A baby at 32 weeks or above has a fairly regular cycle of sleep and wakefulness. It may have learned to suck its thumb too. If its thumb pops out of its mouth, its head darting from side to side may be felt as it tries to find it again. Ten movements within in two hours should be felt normally [8].

In the ninth month, these rapid-fire pummeling kicks appear even less as there's just no room for that. But as the baby turns over, it would definitely get the mother's attention. When the baby engages or drops headfirst down into the pelvis some time before delivery, such as two to three weeks before the due date, the activity patterns may change again. Activities during the last few weeks before delivery are highly dependent on the individual babies some move a bit less, while a few may surprise their mothers with energetic paces before the face to face introduction. These movements are categorized as startle, general body movements, hiccups, breathing movements, isolated arm and leg movements and colonic movements [9].

An unexpected sound from outer surface of the womb would cause the fetus to bend laterally in the direction from which the sound comes. These primitive motor patterns arise in the fluid uterine fluid environment [10]. That is the reason why, in some prenatal sound stimulation programs, the extensive use of only rhythmical, and melodic compositions can be seen [11] since loud and spectrally ugly stimulus would be unethical to reduce the fetus. Moreover, according to the definition of startle reflex of the fetus, it tends to move away from the stimulus. As a behavioral aspect it may be taken as that baby would try to get closer to pleasant sounding signals and away from unpleasant sounding signals. It is really difficult to identify what is pleasant or unpleasant for the baby in the womb. No clear findings are available about this particular area of fetal behavior. The baby's preference may be somehow associated with the mother's liking or disliking 
or the genetic history of the family; further work is required to make any definite conclusion.

All of these findings motivate us to plan this study. It is aimed to establish the correlation between what is recorded or published after experimental research [12-14] and what is reported on the basis of personal experiences of therapists [11] and mothers attending musical therapies for better fetal behavioral development or improving the malposition of the fetus.

\section{MATERIAL AND METHODS}

Forty six patients with singleton and uncomplicated pregnancies were observed. Subjects were obtained from the rural area of Badeen, Pakistan. Since the study is based on non-invasive method and FDA approved equipment under ALARA guidelines so no IRB was required but still the protocol, its feasibility and safety aspects were discussed with the medical team and Gynecology department of Saifi Hospital, Karachi, Pakistan. After full consent of qualified personnel we perform the experiments. The portable ultrasound machine was rented. Fetal movement monitor and sound level meter were the courtesy of an equipment supplier. All patients volunteered willingly. This study was carried out with the help and assistance of a Medical Camp run by a local NGO especially for full-term pregnant women. Patients with body mass index (BMI) of 18.5 to 23 were chosen. The gestational ages were in the range of 36-42 (+/-1) weeks. All of the subjects delivered normally with no physical and neurological fetal abnormities.

The patients were observed with real-time portable Ultrasound Scanner (GE LOGIQ e®) with a $5 \mathrm{MHz}$ frequency and pulse repetition frequency was kept greater than $8 \mathrm{KHz}$ to eliminate the inherent sound stimulus associated with pulse wave Doppler mode [15]. A fetal movement monitor (Philips, M2702A Antepartum Avalon FM20) was also used to precisely measure the fetal movements taking account of the variations in fetal heart rate. Sony DFJ041 Walkman CD player was used to play the music. To reduce ambient noise interference, MDR-J0Z1 clip-style headphones were placed inside a rubber tubing of oxygen mask. The length of tube was 1.25 meters.

A stimulus was designed using four different musical instruments that included the flute, piano, kick drum and bass guitar. The stimulus is designed is in the form of pulsed signal. The sequence of each instrumental can be seen in Fig. 1.

The composition of stimulus 1 is first described. The horizontal axis is timed in seconds and the width of each bar is 10 seconds. The gaps between the bars are 5 seconds long. Stimulus 2 is slightly different in terms of its duty cycle. "On" duration of each segment of instrument is increased from 10 seconds to 15 seconds and "off" time is reduced to 5 seconds. It is done since stimulus 2 has to be given away from the head and short duration stimulus may not be enough to be acknowledged by the fetus, in this scenario. It can also be tested that whether duration affects the response? The total duration of each stimulus is 4 minutes. The SPL was maintained around $70-90 \mathrm{~dB}$ when measured at the opening of oxygen mask, considering intra uterine attenuation of sound signal similar to [1].

The total duration of the patient exposure to the ultrasound during the complete procedure was 40 minutes. The patient was observed for 10 minutes to record pre-stimulus data and head position was marked by an " $\mathrm{X}$ " on the mother's

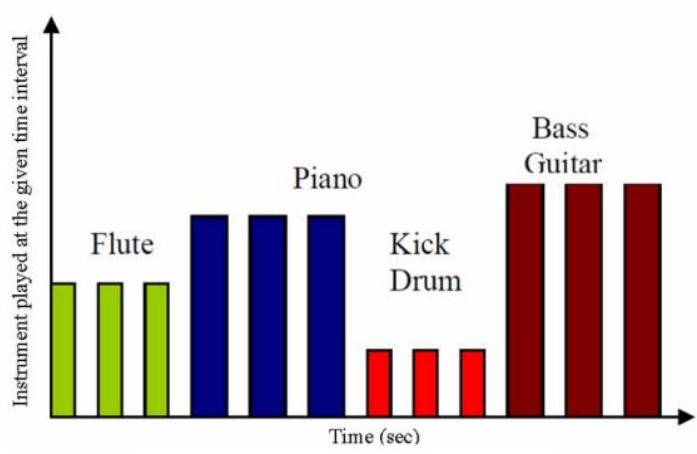

Fig 1. Instrumental sequence of stimulus

abdomen, then the oxygen mask was placed near the head with the mother lying with a straight back in a comfortable position. Stimulus 1 was then applied to measure the average and maximum change observed in FHR and the type of instrument that caused the maximum change. Random movements of short duration and weak intensity were ignored [16], but sustained / strong and short/ strong movements were counted. The same stimulus was played again for 4 minutes. After an interval of 4 minutes, stimulus 2 was applied 4-6 inches away from the head to record the same parameters. If displacement in the head position was reported by the sonographer, then the new head position was marked as the head stopped moving. That displacement is then measured taking umbilicus [17].

An interval of four hours was inserted between stimulus and post-stimulus examination. The recorded data then plotted using Matlab and Bartlett's test and one tailed t-test were performed on SPSS. The test was suggested by the Statistician and level of significance is kept like for greater $p$-values $(p>$ 0.5 ) of $t$-test the hypothesis is rejected. Bartlett's test is to confirm the distribution followed by the group under test. A low $p$ value is for the non-Gaussian data or unequal variances. 


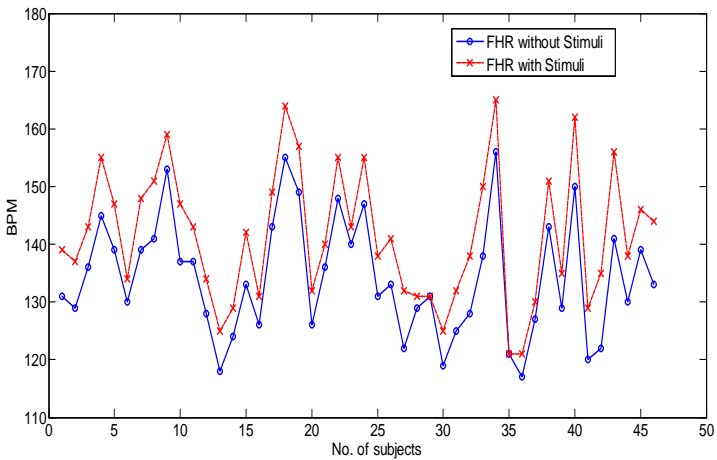

Fig 2. Pre-stimulus and post stimulus fetal heart rate in BPM

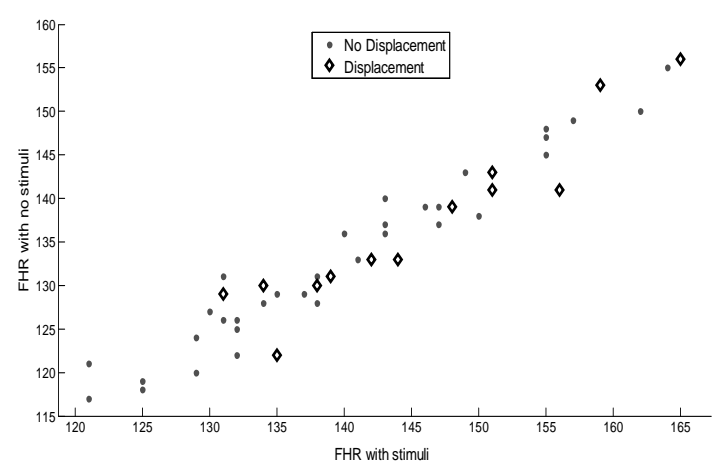

Fig 3. Pre-stimulus and post stimulus fetal heart rate values with successful and unsuccessful output indicators

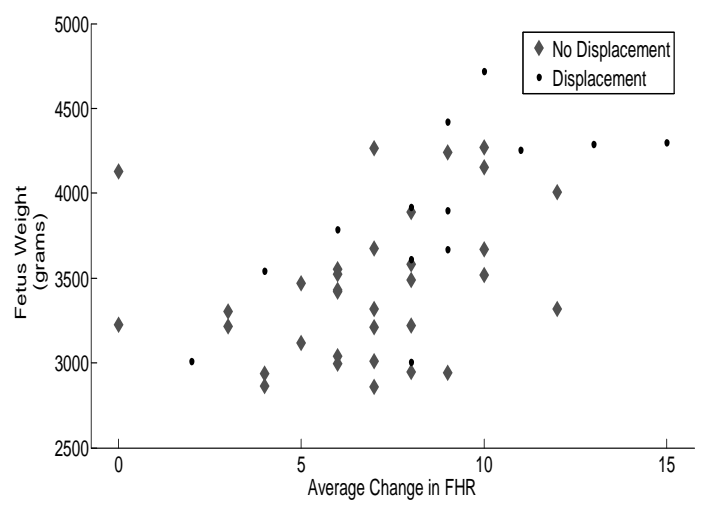

Fig 4. Scatter plot of average change in fetal heart rate versus the fetal weight in grams

\section{RESULTS \& DISCUSSION}

Out of the 46 subjects there were 32 female and 14 male fetuses. Thirteen showed displacement when stimulus was moved away from the head. The magnitude of the displacement was not calculated as it was of secondary importance with respect to the hypothesis investigated. The percentage of success is 28.2 hence not positive but still helpful to analyze the parametric differences between favorable and unfavorable outcomes in terms of displacement. Figure 2 shows the pre-stimulus and with-stimulus fetal heart rates. The pattern of change is very much in agreement to the pre-stimulus state. That is the average change in fetal heart beat count is consistent with its pre-stimulated count. Figure 3 elaborates the same FHR values with successful and

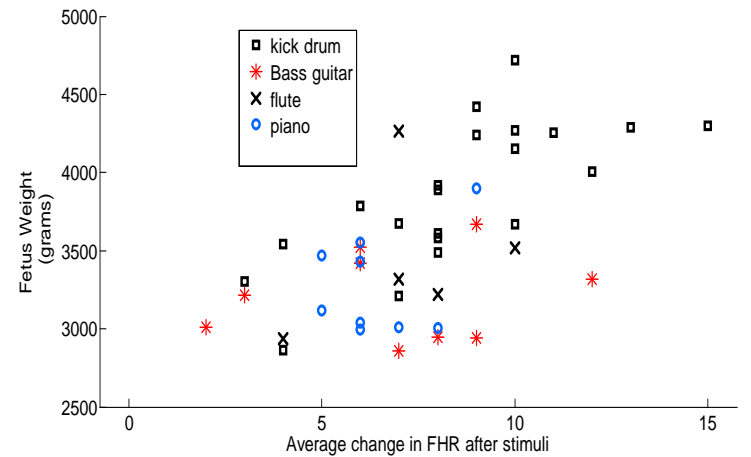

Fig 5. Scatter plot of fetal weight, average change in FHR after stimulus and the type of stimulus that stimulated the maximum beats

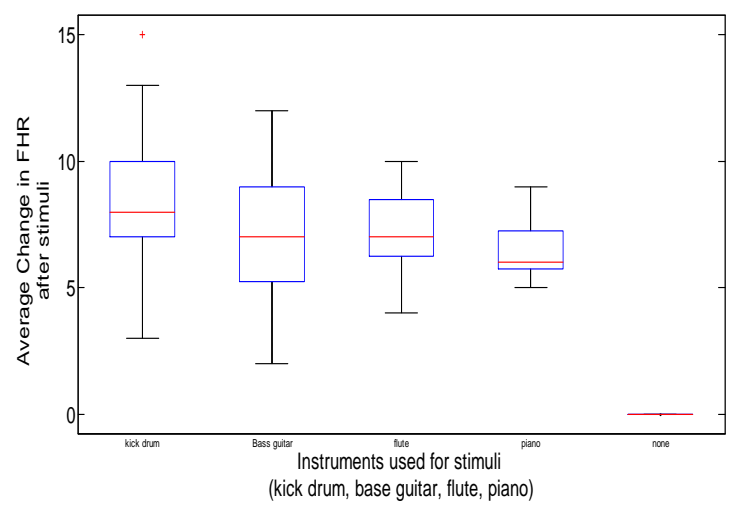

Fig 6. Statistical boxplot shows the average change that an instrument caused to the fetal heart rate

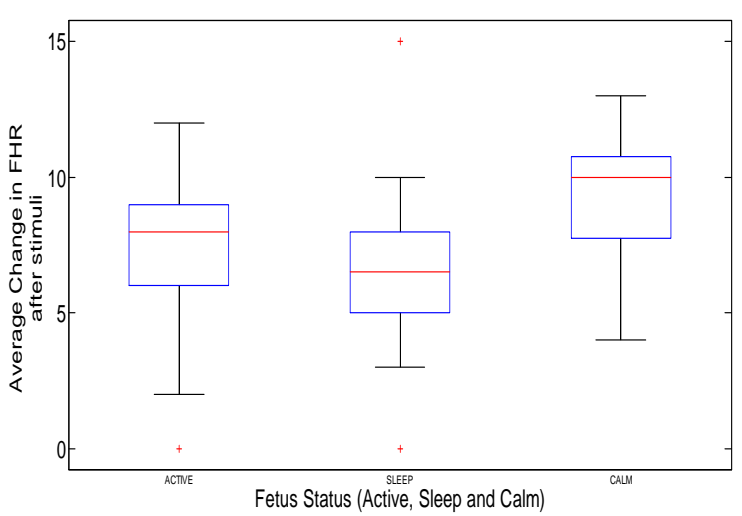

Fig 7. Statistical boxplot shows the pre-stimulus state of fetus against average change in FHR after being stimulated

unsuccessful output indicators. It can be seen clearly that all displacements except one, occurred above the mean value of FHR without stimulus which is $134 \mathrm{bpm}$ (beats per minute) with S.D +/- 10. When comparison was performed on the basis of fetal weight then all cases with positive output ignoring the two exceptions are found to be at or above 3400 gm. with S.D of $512.516 \mathrm{gm}$. Once again it is very close to the mean value of fetal weight as shown in Table 1. Figure 4 is a scatter plot of average change in fetal heart rate versus the fetal weight in grams. The symbol " " indicates the unsuccessful outcomes of the experiment in terms of displacement. 
Table 1. Statistical analysis of foetal basic parameters

\begin{tabular}{|l|c|c|c|}
\hline \multicolumn{1}{|c|}{ Foetal basic parameters } & Mean & $\begin{array}{c}\text { Std. } \\
\text { Deviation }\end{array}$ & N \\
\hline Fetal weight (gm) & 3591.76 & 512.540 & 46 \\
\hline FHR (without stimuli) & 134.22 & 10.169 & 46 \\
\hline $\begin{array}{l}\text { Avg. change in FHR after } \\
\text { stimulus }\end{array}$ & 7.30 & 3.097 & 46 \\
\hline Avg. change after stimulus & 141.52 & 11.471 & 46 \\
\hline $\begin{array}{l}\text { Max. change in FHR after } \\
\text { stimulus }\end{array}$ & 11.46 & 5.054 & 46 \\
\hline
\end{tabular}

Table 2. the detailed statistical analysis shows the average change that an instrument caused to the fetal heart rate

\begin{tabular}{|l|c|c|c|}
\hline \multicolumn{1}{|c|}{ Group } & Count & Mean & Std Dev \\
\hline Kick drum & 21 & 8.57143 & 2.9423 \\
\hline Bass guitar & 9 & 6.88889 & 3.10018 \\
\hline Flute & 5 & 7.200 & 2.16795 \\
\hline Piano & 9 & 6.4444 & 1.3333 \\
\hline Pooled & 46 & 7.30465 & 2.6275 \\
\hline Bartlett's statistic & \multicolumn{3}{|c|}{ Inf } \\
\hline Degree of Freedom & \multicolumn{3}{|c|}{0} \\
\hline p-value & \multicolumn{3}{|c|}{} \\
\hline
\end{tabular}

Table 3 Statistical analysis shows the average change that an instrument caused to the fetal heart rate

\begin{tabular}{|l|l|l|l|}
\hline Group & Count & Mean & Std. Dev \\
\hline Active & 27 & 7.33333 & 2.78733 \\
\hline Sleep & 14 & 6.57143 & 3.52386 \\
\hline Clam & 5 & 9.2 & 3.27109 \\
\hline Pooled & 46 & 7.30435 & 3.07363 \\
\hline Bartlett's Statistics & 0.97622 & \\
\hline Degree of freedom & 2 & \\
\hline p-value & 0.61379 \\
\hline
\end{tabular}

Figure 5 relates the three important primaries of the experiment namely fetal weight, average change in FHR after stimulus and the type of stimulus that stimulated the maximum beats. The scatter plot indicates that kick drum is the highest scorer out of the four. To further investigate the reason for such response the FFT was plot for the kick drum instrumental that was used as the stimulus. Piano and Bass Guitar mostly stimulated the maximum response in fetuses with comparatively low weight of under 4000 grams. Statistical boxplots of Fig. 6 illustrates the range of average change that an instrument caused to the fetal heart rate. The noticeable thing is that the change caused by kick drum is on the higher side. Bass guitar and flute overlaps the bottom range of the kick drum but piano is nowhere in the range that of kick drum. Table 2 shows the detailed statistical analysis of this plot. We observed that every fetus had one of the three states $1 \mathrm{~F}, 2 \mathrm{~F}$ and $4 \mathrm{~F}$ as in [9]. We take $1 \mathrm{~F}$ as sleep, $2 \mathrm{~F}$ as calm and $4 \mathrm{~F}$ as active. Fetus with $2 \mathrm{~F}$ state showed the high range of change in FHR. Figure 7 is the statistical plot of the experiment results showing the pre-stimulus state of fetus against average change in FHR after being stimulated. Two out of 46 subjects did not show any change in FHR after stimulation as shown by red stars at the bottom of the boxplot. The statistical outcomes can be seen in Table 3. Post-stimulus examinations (after $4 \mathrm{hr}$ of experiment) showed a settled response in $100 \%$ of cases.
To investigate the possibility of increasing the success rate of this experiment, a statistical comparison of yes (displaced) and no (not displaced) is done. When success event is observed in terms of type of instrument then kick drum has the highest score followed by bass guitar, and piano as shown in Fig. 8. These values were taken with the help of maximum change occurring in FHR during the procedure and type of stimulus was tracked using FHR charts. No success resulted for flute stimulus. The E4 and F4 notes were played which occupies spectrum between $645 \mathrm{~Hz}$ and $743 \mathrm{~Hz}$. As per the findings of [1] that the required intensity for lower frequencies to make them sensed by the fetus is slightly elevated than for higher frequencies like $1000 \mathrm{~Hz}$ or above. All fetuses showed response to the stimulus but very few actually tend to change their position. But in both cases the kick drums prove to be significant. Boosting frequencies from $100 \mathrm{~Hz}$ to $1 \mathrm{kHz}$ in excess caused the kick drum and bass guitar relationship to be very boomy and muddy and the sequence we played is very much similar to that.

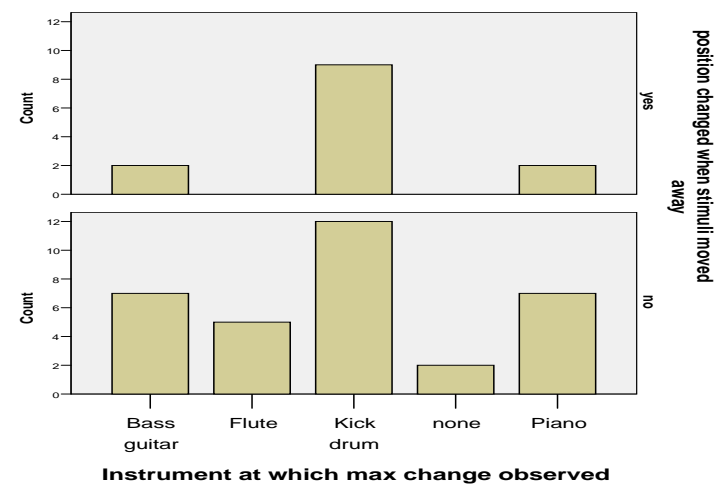

Fig 8. A statistical comparison of yes (displaced) and no (not displaced)

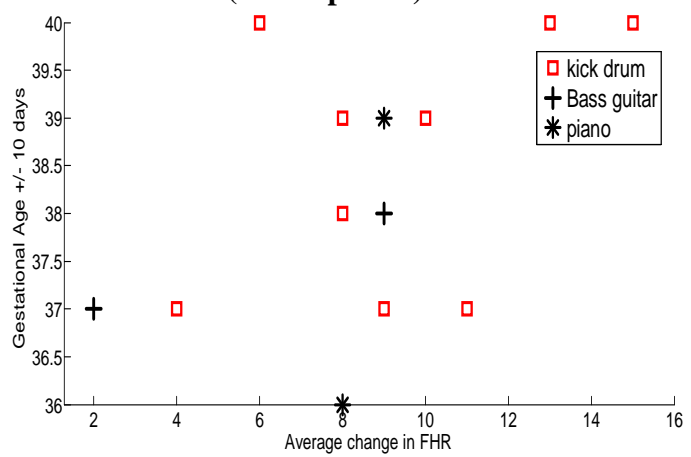

Fig 9. Trend of successful events with respect to fetal gestational age and the musical instrument

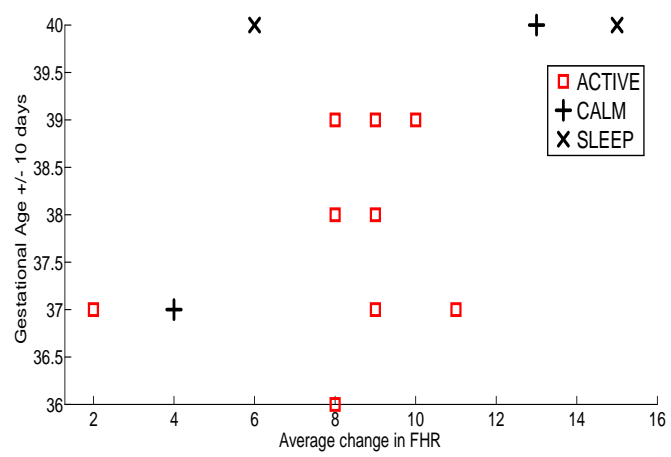

Fig 10. Trend of successful events with respect to fetal gestational age and fetal state 
Figure 9 explains the trend of successful events with respect to fetal gestational age and the musical instrument. Once again it shows that the kick drums are appreciated by almost every age group taken for experiment above 36 weeks, but piano and bass guitar are only effective for those less than 39 weeks. When it comes to fetal state then it is found that most of the active fetuses are found in successful group shown in Fig. 10. Figure 11 shows the number of successes versus the fetal presentation. Almost all cases of oblique presentation resulted in successes. A few (4/13) cephalic babies also tend to move towards the stimulus. The reason for this, which was confirmed by the obstetrician, was that the babies were not trapped in the pelvic brim. Comparing the total number of fetuses with respect to the presentation style, 9 out of the 14 oblique fetuses responded positively in favor of the hypothesis while only 4 out of the 31 cephalic cases were positive. The rate of success is therefore $64.3 \%$ for oblique cases. Of the success cases, $77 \%$ were found to have the gestational age of 36-39. Only 3 cases were of 40 weeks but with oblique presentation showed displacement. The argument supporting the claim that the oblique presentation is more favorable to successes can be accounted for by the fact that it is an uncomfortable position for the full-term fetus, thus causing it to move more easily. The term uncomfortable is used since it is not the usual position at term for a normal delivery. Figure 12 clearly shows that no success for any fetus with GA above 40 weeks. The highest number of successful outcomes resulted for GA of 37 weeks. Out of all 46 patients there were 13 fetuses of 38 weeks, 8 of 37 weeks, 9 of 39 and 40 weeks each, 4 of 41 weeks, 2 of 36 and 1 of 42 weeks gestational age. In "yes" group 4 were found to be 37 weeks that is $50 \%$ of total subjects of the same group. The rest statistics is like $1 / 2$ of 36 weeks, 2/13 of 38 weeks, 3/9 of 39 weeks, and 3/9 of 40 weeks. Although 36 weeks fetuses also responded with $50 \%$ success but due less number of subjects of this age group it may not be taken as a tangible outcome. If we take gender as the controlling variable then, out of 13 positive outcomes 10 were baby boys. Hence the success score for male fetuses is $10 / 13$. To be more decisive about the results, the fluid measurement was also considered and it was found that all "yes" cases were with Amniotic Fluid Index (AFI) of 15 to 19 $\mathrm{cm}$.

Figure 13 is the statistical analysis of all "yes" cases on the basis of gender. Average change in heart rate is greater for male fetuses. The mean FHR is almost equal to the maximum value of change in FHR for a female fetus.

The scatter plot of Fig. 14 shows that male fetuses weight more than female fetuses. Weight correlates positively with responses in terms of change in fetal heart rate. Gender comparison results also supports [18] that the male fetus matures earlier than female.

Overall, responses of the fetuses to the given stimulus was positive (mean FHR pre-stimulus $=133 \mathrm{bpm} /$ mean FHR with-stimulus $=141 \mathrm{bpm}, \mathrm{p}$-value $=0.008)$. The male fetus comparison results in higher $\mathrm{p}$-value $=0.375$ (mean FHR of all cases with stimulus $=141 \mathrm{bpm} /$ mean FHR "yes" male fetuses $=147 \mathrm{bpm}$ ). The GA and fetal weight analysis has also resulted in higher $p$-values around 0.61 . The larger $p$ values simply do not permit to eliminate these primaries to be considered while making conclusions.

\section{CONCLUSION}

This study was to find out if musical instruments can be used as stimuli to move a fetus's head position. One application is to correct the mal-positioning problem in fetuses. While the

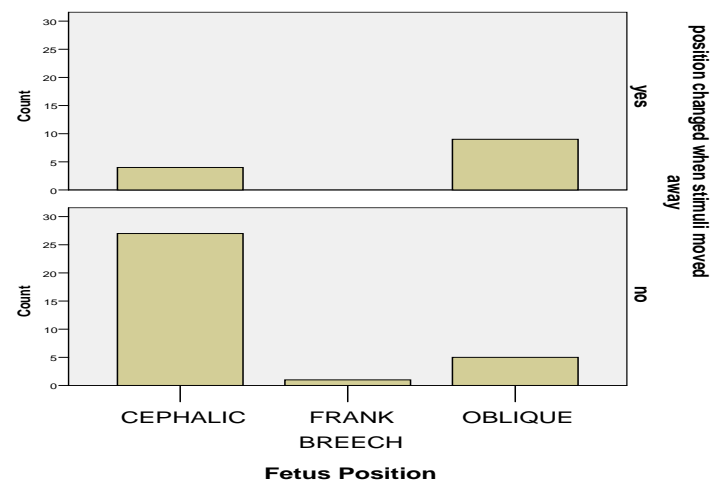

Fig 11. A statistical comparison of yes (displaced) and no (not displaced) versus the fetal presentation

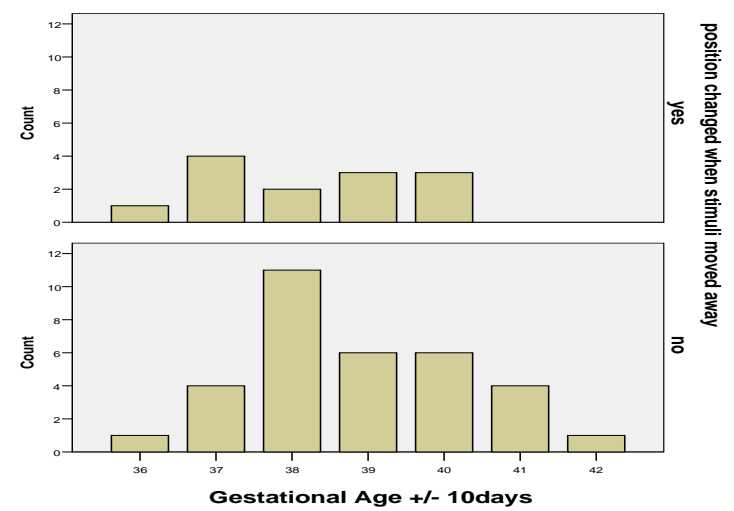

Fig 12. A statistical comparison of yes (displaced) and no (not displaced) versus the gestational age

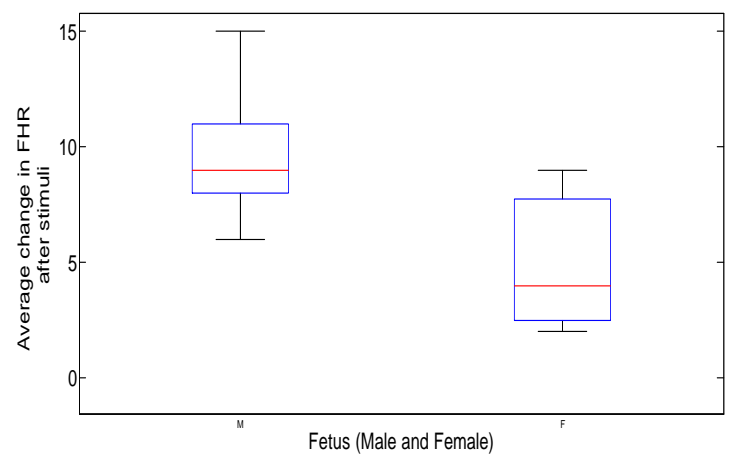

Fig 13. Statistical box plot shows the average change that an instrument caused to the fetal heart rate

success rate is lower than expected, this study examined many factors that could influence the displacement results, and opens door to further study that might improve success rates. The experiment results do not support the hypothesis that musical instruments can consistently elicit head movements from fetuses.

\subsection{Recommendations}

A few recommendations can also be made based on the experiment result like before choosing the instrumental music, the speech spectrum of the mother may also be considered. The position of the mother may also be changed to semi lying or even standing to utilize the gravitational pull for the movement of head towards the exit. To magnify the stimulus, the same piece of music may be played to the mother simultaneously through headphones. Naturally sweet food 


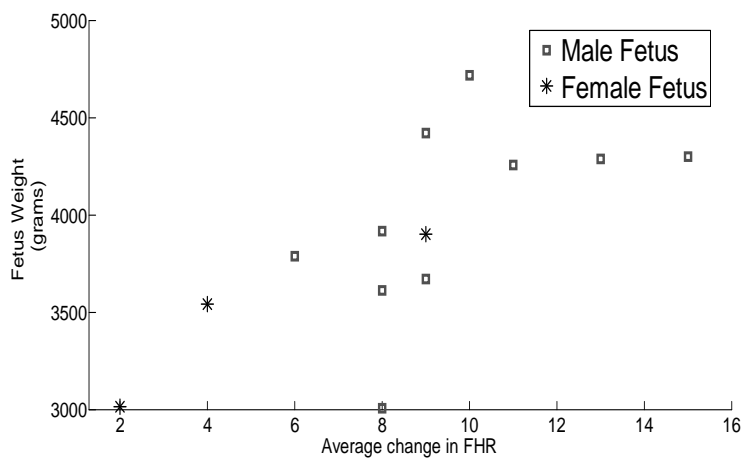

Fig 14. Scatter plot shows the average change to the fetal heart rate versus the fetal weight with gender classification

may be given an hour before to reduce the reaction time. As per the trend of current results, it can be empirically said that with all stated additional measures, a male fetus with oblique presentation, adequate amniotic fluid and gestational age of around $38+/-1.5$ week and fetal weight around $3800-4000$ grams, if stimulated with $1000 \mathrm{~Hz}-1200 \mathrm{~Hz}$ frequency composition mainly produced by kick drum and bass guitar at around $80 \mathrm{~dB}$ SPL may show displacement, when stimulus moved away towards the lower abdomen.

\section{ACKNOWLEDGMENT}

We thank all the participants who volunteered for this study even under the severe catastrophic condition of the flood. We highly acknowledge the efforts of technical and paramedical staff of the medical camp.

\section{CONFLICT OF INTEREST}

The authors declare that no conflicts of interest exist.

\section{REFERENCES}

[1] P.G. Hepper, B.S. Shahidullah, Development of fetal hearing, Archives of Disease in Childhood 71(1994) F81-F87.

[2] L.S. Smith, P.A. Dmochowski, D.W. Mior, B.S. Kisilevsky, Estimated cardiac vagal tone predicts fetal responses to mother's and stranger's voices, Developmental Psychobiology 49(2007) 543 - 547.

[3] N. RM, K. C, M. S, Z. M, Computerized Cardiotocography analysis of fetal heart response to acoustic stimulation Revista Brasileira de Ginecologia e Obstetricia 31(2009) 547 - 541.

[4] Y.J. Park, S.H. Park, Y.J. Kim, J.K. Hoh, Y.S. Park, M.I. Park, Computerized fetal heart rate monitoring after vibroacoustic simulation in the anencephalic fetus, Eary Human Development 86(September 2010) 569-572.
[5] J.P. Newnham, S.E. Burns, B.D. Roberman, Effect of vibratory acoustic stimulation on the duration of fetal heart rate monitoring tests, American Journal of Perinatology 7(1990) 232-234.

[6] J.K. Hoh, Y.S. Park, K.J. Cha, M.I. Park, Fetal heart rate after vibroacoustic stimulation International Journal of Gynaecology and Obstetrics 106(2009) 14-18.

[7] R. Gagnon, C. Hunse, L. Carmichael, F. Fellows, J. Patrick, External vibratory acoustic stimulation near term: fetal heart rate and heart rate variability responses., American Journal of Obstetrics and Gynecology 156(1987 ) 323-327.

[8] K. Picquadio, T. Moore, A prospective evaluation of fetal movement screening to reduce the incidence of antepartum fetal death, Am J Obstet Gynecol. 160(1989) $1075-1080$.

[9] M.D. Velazquez, W.F. Rayburn, Antenatal Evaluation of the Fetus Using Fetal Movement Monitoring, Clinical Obstetrics and Gynecology 45(2002) 993-1004.

[10] M. Jolley, Mind in Motion: A Look into How and Why "The Right Thing Does itself", AmSat News(2009).

[11] Thomas Blum, C. Panthuraamphorn, L. Shihora, Essentials BabyQ (9+9) December 2011, 2008.

[12] H. Eswaran, C.L. Lowery, J.D. Wilson, P. Murphy, H. Preissl, Fetal magnetoencephalography - a multimodal approach, Developmental Brain Research 154 (2005) $57-62$.

[13] P.E. Wilkin, Prenatal and Postnatal Responses to Music and Sound Stimuli: A Clinical Report, Prenatal perception, learning and bonding(1993) 307-329.

[14] R.M. Abrams, Some Aspects of the Fetal Sound Environment, Perception and cognition of music(1995) 83 - 101.

[15] M. Fatemi, A. Alizad, J.F. Greenleaf, Characteristics of the audio sound generated by ultrasound imaging systems, Journal Acoustical Society of America 117(2005) 1448 - 1454.

[16] W.F. Rayburn, Monitoring fetal body movement, Clinical Obstetrics and Gynecology 3(1987) 899 - 911.

[17] Chord, (Math Open Reference, www.mathopenref.com/chord.html).

[18] C. Buss, E. Davis, Q. Class, M. Gierczak, C. Pattillo, L. Glynn, C. Sandman, Maturation of the human fetal startle response: evidence for sex-specific maturation of the human fetus, Early Human Development 85(2009) 688-688. 